\title{
The Current Health Challenges After More than One Year of COVID-19 Pandemic
}

\author{
Farahnaz Khajehnasiri (iD ${ }^{1}$ and Mohsen Poursadeqiyan (ii) ${ }^{2,3, *}$ \\ ${ }^{1}$ Department of Community Medicine, Faculty of Medicine, Tehran University of Medical Sciences, Tehran, Iran \\ ${ }^{2}$ Social Determinants of Health Research Center, Ardabil University of Medical Sciences, Ardabil, Iran \\ ${ }^{3}$ Department of Occupational Health Engineering, School of Health, Ardabil University of Medical Sciences, Ardabil, Iran \\ "Corresponding author: Department of Occupational Health Engineering, School of Health, Ardabil University of Medical Sciences, Ardabil, Iran. Email: \\ poursadeghiyan@gmail.com
}

Received 2021 August 07; Revised 2021 August 13; Accepted 2021 August 13.

Keywords: Health, COVID-19, Occupational Health, Work, Health Life, Quality of Life

\section{Dear Editor,}

The severe acute respiratory syndrome new corona virus 2 (SARS-CoV-2) has spread with remarkable speed, and it was announced as a global health problem by the World Health Organization (WHO) more than one year ago $(1,2)$. there is no effective and specific treatment for the disease due to the different variants of the SARS-COV-2 so far. Therefore, the only way to deal with this virus is a low-cost and effective way of prevention (1). Among prevention methods, the observation of disinfection principles, use of personal protective equipment in normal people and employees at risk, hygienic and safe disposal of contaminated waste, provision of mental health, and stress management can be mentioned $(1,3,4)$. After over one year of the pandemic, there are still many unanswered questions and ambiguities about the pandemic in societies. These questions can further draw the attention of officials to the need to raise the level of awareness of the general population and employees and change their behavior and lead to the development of the use of methods to prevent and deal with the virus effectively (1).

Among the factors causing this disease to become a long-term pandemic are behavioral factors such as nonobservance of social distance due to cultural reasons, holding parties and gatherings, non-observance of home quarantine of patients, the inevitability of travel, and the use of public transportation. These factors increased the number of patients and the inevitability of full protection of medical staff due to high exposure. One of the consequences of this situation is an increase in the frequency of infections and deaths among medical staff and their families (5). Pandemic prolongation became one of the effective factors in the non-observance of preventive behaviors. People are tired of the current situation and cannot tolerate the continuation of restrictions, and as a result, they have lost their sensitivities regarding the observance of prevention strategies (6). The most important danger at this time is the normalization of protective measures. In fact, this characteristic, which has mostly a psychological aspect, causes more dangerous events in society (4). Organizational and national decisions are another challenge. In this case, we may cite the following issues: lack of enforcing strict laws such as mandatory use of masks; lack of coordination of relevant organizations and institutions in disease control; lack of financial support for businesses to comply with home quarantine; lack of optimal protection in the medical staff; and lack of necessary arrangements for access to vaccines (7). Besides, due to the uncertainty of the duration of this pandemic, the hope of eradicating the disease has led to the lack of a long-term plan to telecommuting in work and education places in some countries.

Among biological factors in the cognition and screening of the patients, we may mention the difference in occurrence of various symptoms in each person and the different incubation period, which can disrupt the process of diagnosis. The restrictions on conducting any studies in the real world are due to the danger of the virus. More than one year after the pandemic, the exact duration of virus suspension (indoors and outdoors) has not been determined yet. For example, while the WHO reported airborne transmission of the coronavirus as eight hours (8), the American Institute for Allergy and Infectious Diseases Research stated that the coronavirus was alive three hours after spreading through tiny cough droplets or sneezes (9). The effective dose of the virus to get sick, the length of the 
immune period after receiving the vaccine, new mutations of the virus, and the possibility of re-infection are some of the questions that are not definitively answered yet. Challenges related to the lack of access to screening tests in many cities in developing countries, which calls for social justice despite the global epidemic of the disease, and the high percentage of false-negative errors in screening tests are other problems.

Regarding the challenges related to the efficiency and effectiveness of preventive devices and measures, issues such as a lack of comprehensive studies on the extent of protection by mask in the indoor environment, the type of mask with appropriate performance, the optimal time of protection by the mask, and confusion in the use or nonuse of gloves as a preventive measure can be addressed. Another problem is the uncertainty of scientific results about the risk of transmission from surfaces, food, suitable disinfectants, and the time required for disinfection. In the field of prevention, the lack of a proper attitude towards the use of vaccines in each country based on the presented results or rumors in this regard slows down the process of this important preventive measure. In addition, the importance of many other preventive measures, such as indoor air conditioning in the hot and cold seasons of the year, due to the discussion of energy consumption and weather considerations, is still low after one year of the pandemic.

As a result, the improvement of awareness, attitude, and appropriate action towards preventive measures in the general population and employees through making collective efforts should be taken to end this pandemic as soon as possible (10). People in the community should be informed that the transmission of the disease by asymptomatic carriers is also possible and should follow the health protocols in dealing with these people. It is also possible to hold long-term awareness-raising programs for all age groups and occupations by providing prevention education through radio, television, press, and cyberspace. The application of prevention requirements in recreational, educational, and occupational places, as well as economic support by governments should not be disregarded. In addition, monitoring these cases over time can help optimally control the epidemic.

\section{Footnotes}

Authors' Contribution: It was not declared by the authors.

Conflict of Interests: There was no conflict of interest. Funding/Support: It was not declared by the authors.

\section{References}

1. Arefi MF, Poursadeqiyan M. A review of studies on the COVID19 epidemic crisis disease with a preventive approach. Work. 2020;66(4):717-29. doi:10.3233/WOR-203218. [PubMed: 32925133].

2. Poursadeqiyan M, Bazrafshan E, Arefi MF. Review of environmental challenges and pandemic crisis of Covid-19. J Educ Health Promot.2020;9:250.doi:10.4103/jehp.jehp_420_20.[PubMed:33224994]. [PubMed Central: PMC7657409].

3. Soltaninejad M, Babaei-Pouya A, Poursadeqiyan M, Feiz Arefi M. Ergonomics factors influencing school education during the COVID-19 pandemic: A literature review. Work. 2021;68(1):69-75. doi: 10.3233/WOR-203355. [PubMed: 33427709].

4. Feiz Arefi M, Babaei-Pouya A, Poursadeqiyan $M$. The health effects of quarantine during the COVID-19 pandemic. Work. 2020;67(3):523-7. doi: 10.3233/WOR-203306. [PubMed:33164969].

5. Pasha DF, Lundeen A, Yeasmin D, Pasha MK. An analysis to identify the important variables for the spread of COVID-19 using numerical techniques and data science. Case Studies in Chemical and Environmental Engineering. 2021;3. doi: 10.1016/j.cscee.2020.100067.

6. Ip VHY, Sondekoppam RV. The need to protect health care workers is unchanged in the presence of COVID-19 pandemic fatigue: The probable might still be possible. Anesth Analg. 2021;132(3):e42-3. doi: 10.1213/ANE.0000000000005337. [PubMed: 33591100].

7. Poursadeqiyan M, Feyz Arefi M. Preventive approach and preparation for crisis management before the outbreak. $H D Q .2020$;5(4):165-8. doi: 10.32598/hdq.5.4.189.18.

8. Han Q, Lin Q, Jin S, You L. Coronavirus 2019-nCoV: A brief perspective from the front line. J Infect. 2020;80(4):373-7. doi: 10.1016/j.jinf.2020.02.010. [PubMed: 32109444]. [PubMed Central: PMC7102581]

9. Munster VJ, Koopmans M, van Doremalen N, van Riel D, de Wit E. A novel coronavirus emerging in China - Key questions for impact assessment. N Engl J Med. 2020;382(8):692-4. doi: 10.1056/NEJMp2000929. [PubMed: 31978293].

10. Khajehnasiri F, Zaroushani V, Poursadeqiyan M. Macro ergonomics and health workers during the COVID-19 pandemic. Work. 2021;69(3):713-4. doi: 10.3233/WOR-210412. [PubMed: 34180448]. 